\title{
Non-Bloch-Band Collapse and Chiral Zener Tunneling
}

\author{
S. Longhiø* \\ Dipartimento di Fisica, Politecnico di Milano and Istituto di Fotonica e Nanotecnologie del Consiglio Nazionale delle Ricerche, \\ Piazza L. da Vinci 32, I-20133 Milano, Italy \\ and IFISC (UIB-CSIC), Instituto de Fisica Interdisciplinar y Sistemas Complejos-Palma de Mallorca E-07122, Spain
}

(Received 3 November 2019; accepted 23 January 2020; published 13 February 2020)

\begin{abstract}
Non-Bloch-band theory describes bulk energy spectra and topological invariants in non-Hermitian crystals with open boundaries, where the bulk eigenstates are squeezed toward the edges (skin effect). However, the interplay of non-Bloch-band theory, skin effect, and coherent Bloch dynamics is so far unexplored. In two-band non-Hermitian lattices, it is shown here that collapse of non-Bloch bands and skin modes deeply changes the Bloch dynamics under an external force. In particular, for resonance forcing non-Bloch-band collapse results in Wannier-Stark ladder coalescence and chiral Zener tunneling between the two dispersive Bloch bands.
\end{abstract}

DOI: 10.1103/PhysRevLett.124.066602

Introduction.-Bloch band theory is the fundamental tool to describe electronic states in crystals [1]. Under the action of a weak dc electric field, Bloch theory predicts that electrons undergo an oscillatory motion, the famous Bloch oscillations (BOs) [1], which can be explained from the formation of a Wannier-Stark (WS) ladder energy spectrum [2]. Transitions among different bands occur for stronger fields because of Zener tunneling (ZT) [3]. BOs and ZT are ubiquitous phenomena of coherent wave transport in periodic media and have been observed in a wide variety of physical systems [4-14]. It is remarkable that, after one century from the seminal paper by Felix Bloch [1], there are still treasures to be uncovered within Bloch band theory. For example, Bloch band theory is central in the understanding of topological insulators [15-17] and in the description of flat band systems showing unconventional localization, anomalous phases, and strongly correlated states of matter [18-28]. Recently, a great interest is devoted to extend Bloch band theory and topological order to non-Hermitian lattices [29-67]. In Bloch band theory, an electronic state is defined by the quasimomentum $\mathbf{k}$, which spans the first Brillouin zone, and is delocalized all along the crystal, regardless periodic boundary conditions (PBC) or open boundary conditions (OBC) are assumed. However, in non-Hermitian crystals, something strange happens: energy bands in crystals with $\mathrm{OBC}$ are described by non-Bloch bands that deviate from ordinary Bloch bands, bulk eigenstates cease to be delocalized and get squeezed toward the edges (non-Hermitian skin effect), and the bulk-boundary correspondence based on Bloch topological invariants generally fails to correctly predict the existence of topological zero-energy modes [32-38,44,55, $58,68]$. Recent seminal works $[34,36,44]$ showed that to correctly describe energy spectra and topological invariants in crystals with $\mathrm{OBC}$ one needs to extend Bloch band theory so as the quasimomentum $\mathbf{k}$ becomes complex and varies on a generalized Brillouin zone. Bloch and nonBloch bands can show different symmetry breaking phase transitions and, interestingly, band flattening near an exceptional point (EP) can be observed for non-Bloch bands, while ordinary Bloch bands remain dispersive $[37,55,65]$. As BOs and ZT in non-Hermitian lattices have been investigated in some recent works [69-74], the implications of non-Bloch band theory to particle Bloch dynamics remain obscure. In this Letter we consider Bloch dynamics in a two-band non-Hermitian crystal and unveil a dynamical behavior unique to non-Hermitian crystals: ZT between dispersive Bloch bands, induced by resonance forcing, becomes chiral at the non-Bloch-band collapse point; i.e., electrons irreversibly tunnel from one dispersive Bloch band to the other one, contrary to Hermitian systems where ZT is reversible (oscillatory). Chirality of ZT can be regarded as the signature of non-Bloch-band collapse.

Non-Bloch-band collapse.-We consider a onedimensional tight-binding lattice with chiral symmetry and with two sites per unit cell, driven by an external dc force $F$. In the Wannier basis representation, the Schrödinger equation describing the evolution of the occupation amplitudes $a_{n}$ and $b_{n}$ in the two sublattices $A$ and $B$ at the $n$th unit cell reads

$$
\begin{aligned}
& i \frac{d a_{n}}{d t}=\sum_{l=-q}^{q} \rho_{l} a_{n-l}+\sum_{l=-q}^{q} \theta_{l} b_{n-l}-F n a_{n}, \\
& i \frac{d b_{n}}{d t}=\sum_{l=-q}^{q} \varphi_{l} a_{n-l}-\sum_{l=-q}^{q} \rho_{l} b_{n-l}-F n b_{n},
\end{aligned}
$$

where $\rho_{n}, \theta_{n}$, and $\varphi_{n}$ describe the hopping amplitudes and where we assume that electrons can hop up to the 
$q$ th nearest unit cells. A Hermitian crystal corresponds to $\rho_{-n}=\rho_{n}^{*}$ and $\theta_{-n}=\varphi_{n}^{*}$. For an undriven lattice $(F=0)$, the Hamiltonian in Bloch basis representation reads

$$
H(k)=\sigma_{x} d_{x}(k)+\sigma_{y} d_{y}(k)+\sigma_{z} d_{z}(k),
$$

where $\sigma_{x, y, z}$ are the Pauli matrices and $d_{x}(k) \equiv(1 / 2)$ $\sum_{n}\left(\theta_{n}+\varphi_{n}\right) \exp (-i k n), \quad d_{y}(k) \equiv(1 / 2 i) \sum_{n}\left(\varphi_{n}-\theta_{n}\right)$ $\exp (-i k n), d_{z}(k) \equiv \sum_{n} \rho_{n} \exp (-i k n)$. The energy spectrum shows chiral symmetry $E \leftrightarrow-E$ with the dispersion curves of the two bands given by

$$
E_{ \pm}= \pm \sqrt{Q(\beta)}
$$

where $\beta \equiv \exp (-i k)$ and $Q(\beta) \equiv d_{x}^{2}+d_{y}^{2}+d_{z}^{2}$. Note that $Q(\beta)$ is given by a sum of powers of $\beta$, namely

$$
Q(\beta)=\sum_{l, n=-q}^{q}\left(\rho_{l} \rho_{n}+\theta_{l} \varphi_{n}\right) \beta^{n+l}=P_{2 q}(\beta)+\frac{R_{2 q-1}(\beta)}{\beta^{2 q}}
$$

where $P_{2 q}$ and $R_{2 q-1}$ are two polynomials of $\beta$ of order $2 q$ and $2 q-1$, respectively. Owing to the non-Hermitian skin effect, the energy bands and corresponding eigenfunctions for a long crystal, comprising $N$ unit cells, are different for $\mathrm{PBC}$ and $\mathrm{OBC}[34,44]$. For PBC, $k$ spans the first Brillouin zone $-\pi \leq k<\pi, \beta=\exp (-i k)$ varies on the unit circle $C_{\beta}$ in complex plane, i.e., $|\beta|=1$, and the energy curves (4) describe the ordinary lattice Bloch bands. We assume that the two Bloch bands are separable and $Q(\beta) \neq 0$ in the interior of $C_{\beta}$, so that $E_{ \pm}(k+2 \pi)=E_{ \pm}(k)$. For a lattice with $\mathrm{OBC}$, the energy spectrum is obtained again from Eqs. (4) and (5) but with $\beta$ varying on a generalized Brillouin zone $\tilde{C}_{\beta}$ in complex plane $[34,44]$. The corresponding curves $E_{ \pm}(\beta)$ describe so-called non-Bloch bands, and the bulk eigenstates are squeezed toward the left or right lattice edges depending on whether $|\beta|>1$ or $|\beta|<1$. The generalized Brillouin zone $\tilde{C}_{\beta}$ and corresponding non-Bloch energy bands are obtained from the implicit relation [44]

$$
\left|\beta_{2 q}(E)\right|=\left|\beta_{2 q+1}(E)\right|,
$$

where, for a given complex energy $E, \beta_{l}(E)$ are the roots of the algebraic equation

$$
\beta^{2 q}\left\{P_{2 q}(\beta)-E^{2}\right\}+R_{2 q-1}(\beta)=0,
$$

ordered such that $\left|\beta_{1}(E)\right| \leq\left|\beta_{2}(E)\right| \leq, \ldots, \leq\left|\beta_{4 q}(E)\right|$ [44]. A remarkable property of non-Hermitian lattices is that one can observe non-Bloch flat bands; i.e., $Q(\beta)$ is independent of $\beta$ as $\beta$ varies on the generalized Brillouin zone $\tilde{C}_{\beta}$, while the ordinary Bloch bands remain dispersive; i.e., $Q(\beta)$ is not constant when $\beta$ varies on the unit circle $C_{\beta}$. In particular, it can be shown [75] that, whenever the hopping terms $\rho_{n}, \theta_{n}$ and $\varphi_{n}$ become small and vanish for any $n<0$ (or likewise for any $n>0$ ), the two non-Bloch bands become flat around the two values $E_{ \pm}= \pm E_{0}$, where we have set

$$
E_{0} \equiv \sqrt{\rho_{0}^{2}+\varphi_{0} \theta_{0}}
$$

Correspondingly, the generalized Brillouin zone $\tilde{C}_{\beta}$ shrinks toward the point $\beta=0$ (or $1 / \beta=0$ ); i.e., the collapse of the non-Bloch bands is associated with the coalescence of the skin modes, indicating that at the band collapse the OBC Hamiltonian has two high-order EPs [75]. An example of non-Bloch-band collapse is shown in Fig. 1 for a lattice model which is a variant of the Hamiltonian earlier introduced in Ref. [34] (see also [37,55,68]). The model corresponds to the following nonvanishing values of couplings [Fig. 1(a)]: $\rho_{0}=\Delta, \theta_{0}=\varphi_{0}=t_{0}, \theta_{1}=t+\delta$, and $\varphi_{-1}=t-\delta$, with $\Delta, t_{0}, t$, and $\delta$ real positive numbers. The lattice is Hermitian for $\delta=0$. As $\delta$ is increased, a nonBloch-band collapse at the energies $\pm E_{0}= \pm \sqrt{\Delta^{2}+t_{0}^{2}}$ occurs at $\delta=t$. The generalized Brillouin zone $\tilde{C}_{\beta}$ for this model is a circle of radius $R=\sqrt{(t-\delta) /(t+\delta)}$, which shrinks as $\delta \rightarrow t$ [Fig. 1(c)]. Note that, at the non-Blochbands' collapse point, the ordinary Bloch bands remain dispersive; i.e., band flattening occurs for non-Bloch bands
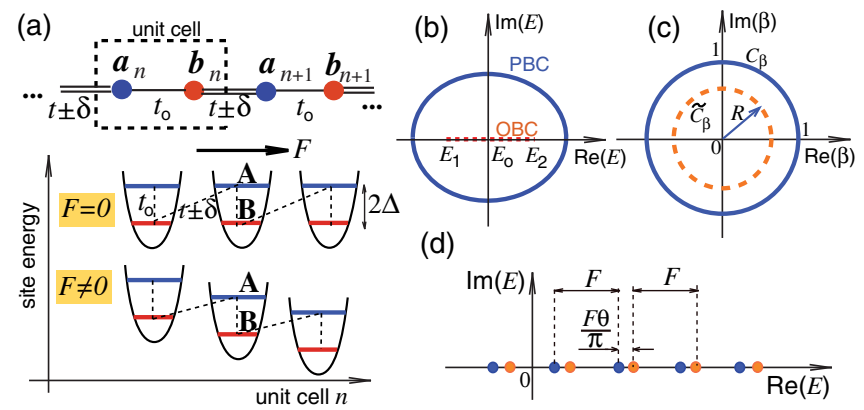

FIG. 1. (a) Example of non-Hermitian lattice showing skin effect. The lattice is Hermitian for $\delta=0$. As $\delta$ is increased, nonBloch-bands collapse at $\delta=t$. An external force $F$ introduces a gradient in the site energies (lower panel). (b) Bloch and nonBloch lattice bands in complex energy plane for $F=0$. The PBC energy spectrum (solid curve) is a closed loop around $E_{0}=$ $\sqrt{\Delta^{2}+t_{0}^{2}}$, whereas the OBC energy spectrum (dashed curve) is the segment $\left(E_{1}, E_{2}\right)$ on the real energy axis, with $E_{1,2}=$ $\sqrt{\Delta^{2}+\left(t_{0} \mp \sqrt{t^{2}-\delta^{2}}\right)^{2}}$. The OBC band collapses to $E_{0}$ at $\delta= \pm t$. Only the positive energy branch $E_{+}(k)$ is shown. (c) Diagram of the Brillouin zone $C_{\beta}$ (unit circle) and generalized Brillouin zone $\tilde{C}_{\beta} . \tilde{C}_{\beta}$ is the circle of radius $R$ which shrinks to $\beta=0$ (skin mode collapse) as $\delta \rightarrow t$. (d) Energy spectrum for a nonvanishing force. The spectrum is entirely discrete (point spectrum) and formed by two interleaved WS ladders. 
solely, which is a distinctive feature than Bloch band flatting in non-Hermitian systems studied in some recent works [76-79].

Chiral Zener tunneling between dispersive Bloch bands. - Let us now consider the case of a driven lattice, i.e., $F \neq 0$. Like for an Hermitian lattice $[80,81]$, the external force changes the energy spectrum from an absolutely continuous spectrum (the two energy bands) into a point spectrum composed by two interleaved WS ladders [75]; see Fig. 1(d). Owing to the formation of the WS ladders, the eigenstates become localized in the bulk. Hence in the driven non-Hermitian lattice the distinction between PBC and OBC smears out and one can disregard boundary conditions assuming that excitation in the lattice remains confined far from the edges. To study ZT between the two dispersive Bloch bands, let us consider wave dynamics in the Bloch basis representation. After setting $a_{n}(t)=\int_{-\pi}^{\pi} d k A(k, t) \exp (i k n)$ and $b_{n}(t)=$ $\int_{-\pi}^{\pi} d k B(k, t) \exp (i k n)$, the evolution equation for the spectral amplitudes $A$ and $B$ reads

$$
i \frac{\partial}{\partial t}\left(\begin{array}{l}
A \\
B
\end{array}\right)=\left(H(k)-i F \frac{\partial}{\partial k}\right)\left(\begin{array}{l}
A \\
B
\end{array}\right) .
$$

The energy spectrum $E^{(\mathrm{WS})}$ of the two interleaved WS ladders can be calculated from Eq. (9) by standard methods and reads (technical details are given in [75])

$$
E_{ \pm}^{(\mathrm{WS})}=l F \pm \frac{F \theta}{2 \pi},
$$

$(l=0, \pm 1, \pm 2, \ldots)$, where $\theta=\theta(F)$ is a complex angle such that $\cos \theta$ is the half trace of the $2 \times 2$ ordered exponential matrix

$$
U=\int_{-\pi}^{\pi} d k \exp \{-i H(k) / F\}
$$

Here we are mainly interested in the case where the angle $\theta$ is real, so that the energy spectrum $E_{ \pm}^{(\mathrm{WS})}$ is real (despite the Hamiltonian is not Hermitian). This case occurs rather generally when $\rho_{0}$ is a real number (positive for the sake of definiteness) and the couplings $\left|\rho_{l}\right|(l \neq 0),\left|\varphi_{l}\right|$, and $\left|\theta_{l}\right|$ are much smaller than $\rho_{0}$. In this limiting case the two weakly dispersive Bloch bands of the lattice describe closed loops around $\pm E_{0} \simeq \pm \rho_{0}$ and correspond to particle occupation mostly in the sublattice $A$ (for the "higher" energy band $E_{+}$) and in sublattice $B$ (for the other "lower" energy band $E_{-}$). In the weak forcing limit $F \rightarrow 0$, an approximate expression of $\theta$ can be derived by a standard WKB analysis and reads

$$
\theta \simeq \frac{1}{F} \int_{-\pi}^{\pi} d k E_{+}(k)=\frac{2 \pi}{F} \sqrt{\sum_{l}\left(\rho_{l} \rho_{-l}+\varphi_{l} \theta_{-l}\right)} .
$$

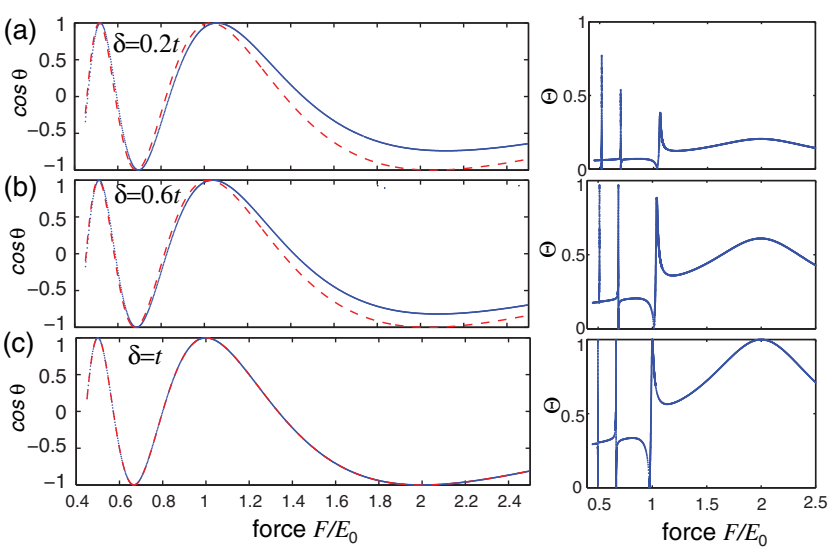

FIG. 2. Left panels: Behavior of $\cos \theta$ vs normalized force $F / E_{0}$ in the driven lattice of Fig. 1 for a few increasing values of $\delta / t$ : (a) $\delta / t=0.2$, (b) $\delta / t=0.6$, (c) $\delta / t=1$ (non-Bloch-band collapse). Other parameters are $\Delta / t=2, t_{0} / t=0.4$. The dashed curves show the behavior of $\cos \theta$ vs $F / E_{0}$ predicted by the WKB analysis. Right panels: behavior of the scalar product $\Theta$ vs $F / E_{0}$.

As shown below, such a result turns out to be exact for any strength $F$ of forcing at the non-Bloch-band collapse, i.e., when $\rho_{l}=\theta_{l}=\varphi_{l}=0$ for $l<0$ (right unidirectional hopping) or for $l>0$ (left unidirectional hopping), so as $\theta=2 \pi E_{0} / F$. Figure 2 shows an example of the numerically computed behavior of the angle $\theta$ vs $F$ for the lattice model of Fig. 1(a) and for increasing values of parameter $\delta$ until non-Bloch-band collapse is attained. The dashed curves in the figure correspond to the approximate result obtained from the WKB analysis. Note that at nonBloch-band collapse point the WKB analysis becomes exact [Fig. 2(c)]. An interesting case is observed when $\theta=0, \pi$, corresponding to the coalescence of the two WS ladders $E_{+}^{(\mathrm{WS})}$ and $E_{-}^{(\mathrm{WS})}$. At the non-Bloch-band collapse point such a coalescence is attained for resonance forcing

$$
F= \pm 2 E_{0} / n
$$

$(n=1,2,3, \ldots)$. While in the Hermitian case such an accidental coalescence yields exact wave packet revivals at time intervals multiplies than $t_{B}=2 \pi / F[80,81]$, in the non-Hermitian lattice the coalescence of energy ladders can correspond to the simultaneous coalescence of the localized WS eigenstates, i.e., to a WS EP [82]. This occurs when the eigenvectors $\mathbf{u}_{1,2}$ of the matrix $U$ [Eq. (11)] become parallel; i.e., when the scalar product $\Theta=$ $\left|\left\langle\mathbf{u}_{1} \mid \mathbf{u}_{2}\right\rangle\right| / \sqrt{\left\langle\mathbf{u}_{1} \mid \mathbf{u}_{1}\right\rangle\left\langle\mathbf{u}_{2} \mid \mathbf{u}_{2}\right\rangle}$ becomes equal to one (right panels in Fig. 2). The main result of this Letter is that (i) a WS EP is observed when the non-Bloch-bands collapse and the external force satisfies the resonance condition (13); (ii) at a WS EP, ZT between the two dispersive Bloch bands becomes chiral; i.e., it ceases to be oscillatory and irreversible tunneling from one dispersive band to the other 
one is observed. To prove such a statement, let us notice that, according the "acceleration theorem" [81,83,84], the external force induces a drift of the Bloch wave number $k$ in time according to $k=k_{0}+F t$ ( $k_{0}$ is the particle quasimomentum at initial time) to cyclically span the Brillouin zone $C_{\beta}$. We then look for an exact solution to Eq. (9) of the form $A(k, t)=f_{A}(t) \delta\left(k-k_{0}-F t\right), B(k, t)=$ $f_{A}(t) \delta\left(k-k_{0}-F t\right)$, and assume without loss of generality $k_{0}=0$. The evolution equation for the amplitudes $f_{A}(t)$ and $f_{B}(t)$ to find the particle in either sublattices $A$ and $B$ reads

$$
i \frac{d}{d t}\left(\begin{array}{c}
f_{A} \\
f_{B}
\end{array}\right)=H(k=F t)\left(\begin{array}{c}
f_{A} \\
f_{B}
\end{array}\right) .
$$

The usual picture of ZT in Hermitian crystals is that Eq. (14) describes a sequence of Landau-Zener transitions between Bloch bands as $k$ crosses the edges of the Brillouin zone, where the band gap is narrower $[80,81,85]$. The resulting quasiperiodic (oscillatory) dynamics is captured by the two Floquet exponents of Eq. (14), which are precisely the terms $\pm F \theta /(2 \pi)$ that define the WS ladder energy spectrum [Eq. (10)]. Note that one can expand $H(k=F t) \quad$ in harmonics as $H(k=F t)=H_{0}+$ $\sum_{l=-q . l \neq 0}^{q} H_{l} \exp (-i l F t)$, where $H_{l}$ are constant $2 \times 2$ matrices, and the eigenvalues of $H_{0}$ are precisely $\pm E_{0}$, given by Eq. (8). When $\rho_{l}=\theta_{l}=\varphi_{l}=0$ for $l<0$; i.e., when the non-Bloch-bands collapse, $H(k=F t)$ is composed solely by negative frequency components for $F>0$, or positive frequency components for $F<0$, so as, under near-resonant forcing, transitions preferentially occur from the "lower" $\left(E_{-}\right)$to the "higher" $\left(E_{+}\right)$energy bands for $F>0$, and viceversa for $F<0[75,86]$. According to the general properties of Floquet systems with one-sided harmonics [87], provided that the force $F$ does not satisfy the resonance condition (13), the two Floquet exponents of Eq. (14) are given by $\pm E_{0}$, the corresponding Floquet eigenstates are linearly independent and the Bloch dynamics is quasiperiodic [75]. This result justifies the exactness of the WKB analysis [Eq. (13)] observed at the non-Blochband collapse point [Fig. 2(c)]. On the other hand, when $F$ satisfies the resonance condition (13), a Floquet EP arises [87]. In this case the dynamics ceases to be oscillatory and an irreversible tunneling from sublattice $B$ (band $E_{-}$) to sublattice $A$ (band $E_{+}$) is observed for $F>0$, but not for $F<0$; i.e., ZT becomes highly asymmetric under force reversal. In other words, chiral ZT is observed because of the appearance of a Floquet EP, which necessarily requires a non-Bloch-band collapse and resonant driving. An example of chiral ZT in the lattice model of Fig. 1(a) is shown in Fig. 3. The figure depicts the dynamical evolution of the amplitudes $a_{n}(t)$ and $b_{n}(t)$ in physical space, norm $P(t)=\sum_{n}\left(\left|a_{n}\right|^{2}+\left|b_{n}\right|^{2}\right)$ and normalized occupation probabilities of the two sublattices $A$ and $B, P_{A}(t)=$ $\left(\sum_{n}\left|a_{n}\right|^{2}\right) / P$ and $P_{B}(t)=\left(\sum_{n}\left|b_{n}\right|^{2}\right) / P$, under resonance (a)

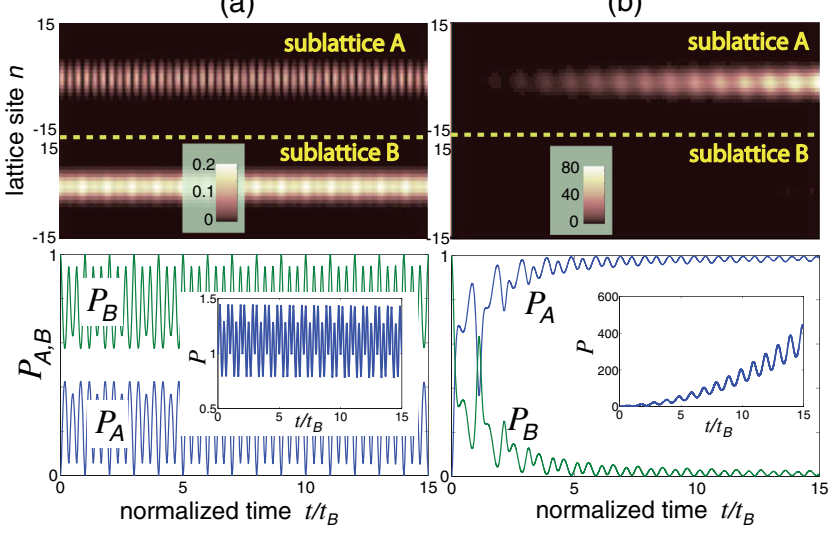

FIG. 3. Chiral ZT in the lattice of Fig. 1 under resonant driving (a) $F=-E_{0}$, and (b) $F=E_{0}$. Parameter values are as in Fig. 2(c). At initial time only sublattice $B$ is excited with a broad Gaussian wave packet of central momentum $k_{0}=0$, i.e., $b_{n}(0) \propto \exp \left[-(n / w)^{2}\right]$ with $w=4$ and $a_{n}(0)=0$. The upper panels show the evolution of $\left|a_{n}\right|^{2}$ and $\left|b_{n}\right|^{2}$ in a pseudo color map, whereas the lower panels depict the evolution of the normalized occupation probabilities $P_{A}$ and $P_{B}$ in the two sublattices $A$ and $B$. The insets show the evolution of the norm $P(t)$. Note that in (a) tunneling into the sublattice $A$ is weak and oscillatory, whereas in (b) irreversible ZT is observed with a secular growth of the norm $P(t)$. Time $t$ is normalized to the BO period $t_{B}=2 \pi / F$.

forcing. The initial condition corresponds to excitation of sublattice $B$. The figure clearly shows that irreversible ZT to sublattice $A$ occurs for $F>0$, but not for $F<0$. In the former case irreversible tunneling is associated to a secular growth of the norm $P(t)$, which is a typical signature of a Floquet EP [87], whereas in the latter case ZT probability is weak and oscillates with the characteristic BO period $t_{B}=2 \pi / F$. For an imperfect non-Bloch band collapse, or for exact non-Bloch-band collapse but nonresonant driving, the tunneling dynamics becomes oscillatory [75]. A simple physical explanation of the appearance of chirality in ZT for the lowest-order $(n=1)$ resonance forcing at the Floquet EP is discussed in [75].

Conclusion.-Non-Bloch-band theory is a powerful tool to describe energy bands and to restore the bulk-boundary correspondence in non-Hermitian crystals showing the skin effect $[34,36,44]$. Here we have reconsidered the old problem of Bloch oscillations and Zener tunneling in the framework of non-Bloch-band theory, and unveiled a major physical effect unique to non-Hermitian systems, namely chiral Zener tunneling between dispersive Bloch bands. This phenomenon is observed under resonance forcing and is rooted in the collapse of non-Bloch bands and skin modes. Such results show major implications of nonBloch-band theory into coherent wave transport and are expected to be of broad relevance in different areas of physics, where wave transport is described by effective non-Hermitian models. 
*stefano.longhi@polimi.it

[1] F. Bloch, über die Quantenmechanik der Elektronen in Kristallgittern, Z. Phys. 52, 555 (1929).

[2] G. H. Wannier, Wave functions and effective Hamiltonian for Bloch electrons in an electric field, Phys. Rev. 117, 432 (1960).

[3] C. Zener, A theory of the electrical breakdown of solid dielectrics, Proc. R. Soc. A 145, 523 (1934).

[4] P. Voisin, J. Bleuse, C. Bouche, S. Gaillard, C. Alibert, and A. Regreny, Observation of the Wannier-Stark Quantization in a Semiconductor Superlattice, Phys. Rev. Lett. 61, 1639 (1988).

[5] J. Feldmann, K. Leo, J. Shah, D. A. B. Miller, J. E. Cunningham, T. Meier, G. von Plessen, A. Schulze, P. Thomas, and S. Schmitt-Rink, Optical investigation of Bloch oscillations in a semiconductor superlattice, Phys. Rev. B 46, 7252(R) (1992).

[6] B. Rosam, D. Meinhold, F. Lser, V. G. Lyssenko, S. Glutsch, F. Bechstedt, F. Rossi, K. Köhler, and K. Leo, Field-Induced Delocalization and Zener Breakdown in Semiconductor Superlattices, Phys. Rev. Lett. 86, 1307 (2001).

[7] M. Ben Dahan, E. Peik, J. Reichel, Y. Castin, and C. Salomon, Bloch Oscillations of Atoms in an Optical Potential, Phys. Rev. Lett. 76, 4508 (1996).

[8] S. R. Wilkinson, C. F. Bharucha, K. W. Madison, Qian Niu, and M. G. Raizen, Observation of Atomic Wannier-Stark Ladders in an Accelerating Optical Potential, Phys. Rev. Lett. 76, 4512 (1996).

[9] O. Morsch, J. H. Müller, M. Cristiani, D. Ciampini, and E. Arimondo, Bloch Oscillations and Mean-Field Effects of Bose-Einstein Condensates in 1D Optical Lattices, Phys. Rev. Lett. 87, 140402 (2001).

[10] R. Morandotti, U. Peschel, J. S. Aitchison, H. S. Eisenberg, and Y. Silberberg, Experimental Observation of Linear and Nonlinear Optical Bloch Oscillations, Phys. Rev. Lett. 83, 4756 (1999).

[11] T. Pertsch, P. Dannberg, W. Elflein, A. Bräuer, and F. Lederer, Optical Bloch Oscillations in Temperature Tuned Waveguide Arrays, Phys. Rev. Lett. 83, 4752 (1999).

[12] S. Longhi, Bloch Oscillations and Zener Tunneling with Nonclassical Light, Phys. Rev. Lett. 101, 193902 (2008).

[13] F. Dreisow, A. Szameit, M. Heinrich, T. Pertsch, S. Nolte, A. Tünnermann, and S. Longhi, Bloch-Zener Oscillations in Binary Superlattices, Phys. Rev. Lett. 102, 076802 (2009).

[14] Y. Zhang, D. Zhang, Z. Zhang, C. Li, Y. Zhang, F. Li, Mi. R. Belic, and M. Xiao, Optical Bloch oscillation and Zener tunneling in an atomic system, Optica 4, 571 (2017).

[15] M. Z. Hasan and C. L. Kane, Colloquium: Topological insulators, Rev. Mod. Phys. 82, 3045 (2010).

[16] A. Bansil, H. Lin, and T. Das, Colloquium: Topological band theory, Rev. Mod. Phys. 88, 021004 (2016).

[17] J. K. Asbóth, L. Oroszlány, and A. Pályi, in A Short Course on Topological Insulators, Lecture Notes in Physics (Springer International Publishing, Switzerland, 2016), Vol. 919.

[18] B. Sutherland, Localization of electronic wave functions due to local topology, Phys. Rev. B 34, 5208 (1986).

[19] E. J. Bergholtz and Z. Liu, Topological flat band models and fractional Chern insulators, Int. J. Mod. Phys. B 27, 1330017 (2013).
[20] O. Derzhko, J. Richter, and M. Maksymenko, Strongly correlated flat-band systems: The route from Heisenberg spins to Hubbard electrons, Int. J. Mod. Phys. B 29, 1530007 (2015).

[21] D. Leykam, A. Andreanov, and S. Flach, Artificial flat band systems: From lattice models to experiments, Adv. Phys. X 3, 1473052 (2018).

[22] J. Vidal, P. Butaud, B. Douot, and R. Mosseri, Disorder and interactions in Aharonov-Bohm cages, Phys. Rev. B 64, 155306 (2001).

[23] S. A. Parameswaran, R. Roy, and S. L. Sondhi, Fractional quantum Hall physics in topological flat bands, C.R. Phys. 14, 816 (2013).

[24] J. D. Bodyfelt, D. Leykam, C. Danieli, X. Yu, and S. Flach, Flatbands Under Correlated Perturbations, Phys. Rev. Lett. 113, 236403 (2014).

[25] R. A. Vicencio, C. Cantillano, L. Morales-Inostroza, B. Real, C. Meijia-Cortes, S. Weimann, A. Szameit, and M. I. Molina, Observation of Localized States in Lieb Photonic Lattices, Phys. Rev. Lett. 114, 245503 (2015).

[26] S. Mukherjee, A. Spracklen, D. Choudhury, N. Goldman, P. Ohberg, E. Andersson, and R. R. Thomson, Observation of a Localized Flat-Band State in a Photonic Lieb Lattice, Phys. Rev. Lett. 114, 245504 (2015).

[27] F. Baboux, L. Ge, T. Jacqmin, M. Biondi, A. Lemaitre, L. Le Gratiet, I. Sagnes, S. Schmidt, H. E. Treci, A. Amo, and J. Bloch, Bosonic Condensation and Disorder-Induced Localization in a Flat Band, Phys. Rev. Lett. 116, 066402 (2016).

[28] R. Khomeriki and S. Flach, Landau-Zener Bloch Oscillations with Perturbed Flat Bands, Phys. Rev. Lett. 116, 245301 (2016).

[29] D. Leykam, K. Y. Bliokh, C. Huang, Y. D. Chong, and F. Nori, Edge Modes, Degeneracies, and Topological Numbers in Non-Hermitian Systems, Phys. Rev. Lett. 118, 040401 (2017).

[30] H. Shen, B. Zhen, and L. Fu, Topological Band Theory for Non-Hermitian Hamiltonians, Phys. Rev. Lett. 120, 146402 (2018).

[31] Z. Gong, Y. Ashida, K. Kawabata, K. Takasan, S. Higashikawa, and M. Ueda, Topological Phases of NonHermitian Systems, Phys. Rev. X 8, 031079 (2018).

[32] Y. Xiong, Why does bulk boundary correspondence fail in some non-Hermitian topological models, J. Phys. Commun. 2, 035043 (2018).

[33] F. K. Kunst, E. Edvardsson, J. C. Budich, and E. J. Bergholtz, Biorthogonal Bulk-Boundary Correspondence in NonHermitian Systems, Phys. Rev. Lett. 121, 026808 (2018).

[34] S. Yao and Z. Wang, Edge States and Topological Invariants of Non-Hermitian Systems, Phys. Rev. Lett. 121, 086803 (2018).

[35] S. Yao, F. Song, and Z. Wang, Non-Hermitian Chern Bands, Phys. Rev. Lett. 121, 136802 (2018).

[36] C. H. Lee and R. Thomale, Anatomy of skin modes and topology in non-Hermitian systems, Phys. Rev. B 99, 201103(R) (2019).

[37] V. M. Martinez Alvarez, J. E. Barrios Vargas, and L. E. F. Foa Torres, Non-Hermitian robust edge states in one dimension: Anomalous localization and eigenspace condensation at exceptional points, Phys. Rev. B 97, 121401(R) (2018). 
[38] V. M. Martinez Alvarez, J. E. Barrios Vargas, M. Berdakin, and L. E. F. Foa Torres, Topological states of non-Hermitian systems, Eur. Phys. J. Spec. Top. 227, 1295 (2018).

[39] A. Ghatak and T. Das, New topological invariants in nonHermitian systems, J. Phys. Condens. Matter 31, 263001 (2019).

[40] K. Kawabata, K. Shiozaki, M. Ueda, and M. Sato, Symmetry and Topology in Non-Hermitian Physics, Phys. Rev. X 9, 041015 (2019).

[41] H. Zhou and J. Y. Lee, Periodic table for topological bands with non-Hermitian Bernard-LeClair symmetries, Phys. Rev. B 99, 235112 (2019).

[42] K. Kawabata, S. Higashikawa, Z. Gong, Y. Ashida, and M. Ueda, Topological unification of time-reversal and particlehole symmetries in non-Hermitian physics, Nat. Commun. 10, 297 (2019).

[43] C.-H. Liu, H. Jiang, and S. Chen, Topological classification of non-Hermitian systems with reflection symmetry, Phys. Rev. B 99, 125103 (2019).

[44] K. Yokomizo and S. Murakami, Non-Bloch Band Theory for Non-Hermitian Systems, Phys. Rev. Lett. 123, 066404 (2019).

[45] T. Liu, Y.-R. Zhang, Q. Ai, Z. Gong, K. Kawabata, M. Ueda, and F. Nori, Second-Order Topological Phases in NonHermitian Systems, Phys. Rev. Lett. 122, 076801 (2019).

[46] L. Herviou, J. H. Bardarson, and N. Regnault, Defining a bulk-edge correspondence for non-Hermitian Hamiltonians via singular-value decomposition, Phys. Rev. A 99, 052118 (2019).

[47] S. Longhi, Loschmidt echo and fidelity decay near an exceptional point, Ann. Phys. (Berlin) 531, 1900054 (2019).

[48] L. Jin and Z. Song, Bulk-boundary correspondence in nonHermitian systems in one dimension with chiral inversion symmetry, Phys. Rev. B 99, 081103(R) (2019).

[49] S. Longhi, Topological Phase Transition in non-Hermitian Quasicrystals, Phys. Rev. Lett. 122, 237601 (2019).

[50] X. Yang, Y. Cao, and Y. Zhai, Non-Hermitian Weyl semimetals: Non-Hermitian skin effect and non-Bloch bulk-boundary correspondence, arXiv:1904.02492.

[51] D. S. Borgna, A. J. Kruchkov, and R.-J. Slager, NonHermitian boundary modes, arXiv:1902.07217v2.

[52] H.-G. Zirnstein, G. Refael, and B. Rosenow, Bulk-boundary correspondence for non-Hermitian Hamiltonians via Green functions, arXiv:1901.11241v1.

[53] F. K. Kunst and V. Dwivedi, Non-Hermitian systems and topology: A transfer matrix perspective, Phys. Rev. B 99, 245116 (2019).

[54] T. S. Deng and W. Yi, Non-Bloch topological invariants in a non-Hermitian domain-wall system, Phys. Rev. B 100, 035102 (2019).

[55] S. Longhi, Probing non-Hermitian skin effect and nonBloch phase transitions, Phys. Rev. Research 1, 023013 (2019).

[56] W. Brzezicki and T. Hyart, Hidden Chern number in onedimensional non-Hermitian chiral-symmetric systems, Phys. Rev. B 100, 161105(R) (2019).

[57] H. Zhao, X. Qiao, T. Wu, B. Midya, S. Longhi, and L Feng, Non-Hermitian topological light steering, Science $\mathbf{3 6 5}$, 1163 (2019).
[58] L. E. F. Foa Torres, Perspective on topological states of nonHermitian lattices, J. Phys. Mater. 3, 014002 (2019).

[59] F. Song, S. Yao, and Z. Wang, Non-Hermitian Skin Effect and Chiral Damping in Open Quantum Systems, Phys. Rev. Lett. 123, 170401 (2019).

[60] F. Song, S. Yao, and Z. Wang, Non-Hermitian Topological Invariants in Real Space, Phys. Rev. Lett. 123, 246801 (2019).

[61] T. Hofmann, T. Helbig, F. Schindler, N. Salgo, M. Brzezinska, M. Greiter, T. Kiessling, D. Wolf, A. Vollhardt, A. Kabasi, C. H. Lee, A. Bilusic, R. Thomale, and T. Neupert, Reciprocal skin effect and its realization in a topolectrical circuit, arXiv:1908.02759v1.

[62] T. Helbig, T. Hofmann, S. Imhof, M. Abdelghany, T. Kiessling, L. W. Molenkamp, C. H. Lee, A. Szameit, M. Greiter, and R. Thomale, Observation of bulk boundary correspondence breakdown in topolectrical circuits, arXiv: $1907.11562 \mathrm{v} 1$.

[63] T. Deng, K. Wang, G. Zhu, Z. Wang, W. Yi, and P. Xue, Observation of non-Hermitian bulk-boundary correspondence in quantum dynamics, arXiv:1907.12566v1.

[64] A. Ghatak, M. Brandenbourger, J. van Wezel, and C. Coulais, Observation of non-Hermitian topology and its bulk-edge correspondence, arXiv:1907.11619.

[65] S. Longhi, Non-Bloch $\mathcal{P} \mathcal{T}$ symmetry breaking in nonHermitian photonic quantum walks, Opt. Lett. 44, 5804 (2019).

[66] J. Y. Lee, J. Ahn, H. Zhou, and A. Vishwanath, Topological Correspondence between Hermitian and Non-Hermitian Systems: Anomalous Dynamics, Phys. Rev. Lett. 123, 206404 (2019).

[67] E. J. Bergholtz, J. C. Budich, and F. K. Kuns, Exceptional topology of non-Hermitian systems, arXiv:1912.10048v1.

[68] T.E. Lee, Anomalous Edge State in a Non-Hermitian Lattice, Phys. Rev. Lett. 116, 133903 (2016).

[69] S. Longhi, Bloch Oscillations in Complex Crystals with $\mathcal{P} \mathcal{T}$-Symmetry, Phys. Rev. Lett. 103, 123601 (2009).

[70] S. Longhi, Bloch oscillations in non-Hermitian lattices with trajectories in complex plane, Phys. Rev. A 92, 042116 (2015).

[71] M. Wimmer, M.-A. Miri, D. Christodoulides, and U. Peschel, Observation of Bloch oscillations in complex $\mathcal{P} \mathcal{T}$-symmetric photonic lattices, Sci. Rep. 5, 17760 (2015).

[72] E. M. Graefe, H. J. Korsch, and A. Rush, Quasiclassical analysis of Bloch oscillations in non-Hermitian tightbinding lattices, New J. Phys. 18, 075009 (2016).

[73] Y.-L. Xu, W. S. Fegadolli, L. Gan, M.-H. Lu, X.-P. Liu, Z.-Y. Li, A. Scherer, and Y.-F. Chen, Experimental realization of Bloch oscillations in a parity-time synthetic silicon photonic lattice, Nat. Commun. 7, 11319 (2016).

[74] B. Longstaff and E.-M. Graefe, Non-adiabatic transitions through exceptional points in the band structure of a $\mathcal{P} \mathcal{T}$ symmetric lattice, Phys. Rev. A 100, 052119 (2019).

[75] See the Supplemental Material at http://link.aps.org/ supplemental/10.1103/PhysRevLett.124.066602 for (i) general proof of non-Bloch-band collapse (Sec. S. 1), (ii) WS ladder formation (Sec. S. 2), (iii) ZT near the collapse point (Sec. S. 3), and (iv) a simple physical picture of chiral ZT (Sec. S. 4). 
[76] H. Ramezani, Non-Hermiticity-induced flat band, Phys. Rev. A 96, 011802(R) (2017).

[77] D. Leykam, S. Flach, and Y. D. Chong, Flat bands in lattices with non-Hermitian coupling, Phys. Rev. B 96, 064305 (2017).

[78] B. Qi, L. Zhang, and L. Ge, Defect States Emerging from a Non-Hermitian Flatband of Photonic Zero Modes, Phys. Rev. Lett. 120, 093901 (2018).

[79] L. Ge, Non-Hermitian lattices with a flat band and polynomial power increase, Photonics Res. 6, A10 (2018).

[80] B. M. Breid, D. Witthaut, and H. J. Korsch, Bloch-Zener oscillations, New J. Phys. 8, 110 (2006).

[81] S. Longhi, Optical Zener-Bloch oscillations in binary waveguide arrays, Europhys. Lett. 76, 416 (2006).
[82] C. Elsen, K. Rapedius, D. Witthaut, and H. J. Korsch, Exceptional points in bichromatic Wannier-Stark systems, J. Phys. B 44, 225301 (2011).

[83] J. Callaway, Quantum Theory of the Solid State (Academic Press, New York, 1974).

[84] V. Grecchi and A. Sacchetti, Acceleration theorem for Bloch oscillators, Phys. Rev. B 63, 212303 (2001).

[85] S. N. Shevchenko, S. Ashhab, and F. Nori, Landau-ZenerStuckelberg interferometry, Phys. Rep. 492, 1 (2010).

[86] S. Longhi and G. Della Valle, Non-Hermitian time-dependent perturbation theory: Asymmetric transitions and transitionless interactions, Ann. Phys. (Berlin) 385, 744 (2017).

[87] S. Longhi, Floquet exceptional points and chirality in nonHermitian Hamiltonians, J. Phys. A 50, 505201 (2017). 